\title{
Philosophiques
}

\section{Des vertus du décentrement dans Les Ruines de Volney}

\section{Jean-Louis Labarrière}

Volume 20, numéro 1, printemps 1993

URI : https://id.erudit.org/iderudit/027206ar

DOI : https://doi.org/10.7202/027206ar

Aller au sommaire du numéro

Éditeur(s)

Société de philosophie du Québec

ISSN

0316-2923 (imprimé)

1492-1391 (numérique)

Découvrir la revue

Citer cet article

Labarrière, J.-L. (1993). Des vertus du décentrement dans Les Ruines de Volney. Philosophiques, 20(1), 113-129. https://doi.org/10.7202/027206ar

\section{Résumé de l'article}

Cette étude analyse comment la mise en scène d'une méditation sur les ruines des anciens empires conduit Volney à opposer le cycle des révolutions à la Révolution française. Les vertus du décentrement, tout autant méthodologiques que philosophiques et politiques, sont multiples et elles permettent d'instruire le procès des religions. Si la Révolution française est exemplaire, c'est donc parce quelle annonce ce mouvement de prise en main de leur destin par les peuples. Pourquoi faut-il dès lors encore recourir à un catéchisme? 


\title{
DES VERTUS DU DÉCENTREMENI DANS IES RUINESDE VOLNEY
}

\author{
par \\ Jean-Louis Labarrière
}

RÉSUMÉ : Cette étude analyse comment la mise en scène d'une méditation sur les ruines des anciens empires conduit Volney à opposer le cycle des révolutions à la Révolution française. Les vertus du décentrement, tout autant méthodologiques que philosophiques et politiques, sont multiples et elles permettent d'instruire le procès des religions. Si la Révolution française est exemplaire, c'est donc parce qu'elle annonce ce mouvement de prise en main de leur destin par les peuples. Pourquoi faut-il dès lors encore recourir à un catéchisme?

ABSTRACT : This study examines how the staging of a meditation on the ruins of the ancient empires leads Volney to contrast the seemingly endless cycle of revolutions to the French Revolution. Decentration has virtues - as much methodological as philosophical and political - that are multiple, and that allow to put religions on trial. If the French Revolution is paradigmatic, it is because it announces this movement by which the peoples will take their destiny in their own hands. But then, why should a catechism still be needed?

Volney publie Les Ruines en I79I, alors que s'achèvent les travaux de l'Assemblée constituante dont il fut l'une des grandes âmes, tant il est vrai qu'à lui aussi s'applique merveilleusement ce compliment adressé par Andrieux à Cabanis : « Vous avez plus d'âme que ceux qui vous accusent de ne pas y croire ${ }^{\mathrm{I}}$. » Après un bref séjour en Corse, peu fructueux en ce qui concerne l'expérience agricole alors tentée, mais intéressant car il y rencontre le

1. Cité dans G. Gusdorf, La conscience révolutionmaire. Les Ideoologues, Paris, Payot, 1978 , p. 343, n. 3 . 
jeune Bonaparte qui le questionne sur l'Égypte en raison du vif succès obtenu par le Voyage en Égypte et en Syrie (i 787 ), Volney publie en 1793 le Catéchisme du Citoyen Français, appelé à devenir le livre second des Ruines sous le titre La Loi Naturelle, ou plus longuement. Principes Physiques de la Morale déduits de l'Organisation de lHomme et de IUnivers.

Un certain Berger, cité par Picavet, parlera plus tard de « l'espèce de délit social dont l'auteur des Ruines et du Catéchisme $d u$ Citoyen Français s'est rendu coupable ${ }^{2}$, délit qui valut à ces livres d'être mis à l'Index en 1821 , soit un an après la mort de Volney. Nul doute que le corps du délit réside en ce fait que Les Ruines sont, disait-on élogieusement peu après leur parution, un

I...I plaidoyer éloquent en faveur des droits des nations, admirable tableau de l'origine et de la filiation des idées religieuses et de l'identité du but des religions, exposé judicieusement exact de la condition de l'homme dans l'univers et des sources des maux des sociétés ${ }^{3}$.

Il est vrai que l'Idéologue Volney y amplifiait le propos du jeune député Volney, qui, en I790, s'opposant à ce que la religion catholique ne devienne religion d'État, écrivait dans son discours une phrase qui allait devenir célèbre grâce à l'emprunt qu'en fit son ami Mirabeau : « Je vois d'ici cette fenêtre, d'où partit l'arquebuse royale qui a donné le signal du massacre de la SaintBarthélemy. ${ }^{4} \gg$

2. Cf. F. Picavet, Les Idéologues, Par is, Alcan, 189I (Olms Reprints, 1972), p. I28.

3. Ce propos est cité dans l'anonyme notice introductive - peut-être de Nicolas David - de l'édition de la Bibliothèque nationale, Collection des meilleurs auteurs anciens et modernes, Paris, 1898 , p. 45. La collection de la Librairie de la Bibliotheque nationale, fondée en r863, avait pour fin de redorer le blason de tous les auteurs ayant concouru au développement de la raison et, par là, à l'avènement de la Révolution française. Le propos cité est laissé anonyme, mais il est très certainement le fait de Guinguené, qui, dans la Décade, loua Volney pour avoir su populariser les thèses établies par Dupuis dans son Origine de tous les cultes ou la Religion universelle (1794). Volney pouvait en connaître la teneur dès le début des années i79o puisque Dupuis en avait publié divers fragments, dont le Mémoire sur l'Origine des Constellations ( $778 \mathrm{I}$ ). Les notes du chapitre XXII des Ruines, comme d'autres longtemps retravaillées et publiées lors d'éditions ultérieures, se réfêrent explicitement aux travaux de Dupuis. Pour d'évidentes raisons de commodité, je me référerai à l'édition en deux tomes des cuvres de Volney due au Corpus des œuures de philosophie en langue française, Paris, Fayard, ig8g (le tome I comprend les Ruines, La Loi Naturelle et les Le. çons dHistoire ; tout ce qui sera souligné dans les citations sera du fait de Volney et non du mien?.

4. Cf. Picavet, op. cit, p. 130. 
Volney n'a donc attendu ni la publication des Ruines ni celle de son Catéchisme pour se rendre coupable de « délit social»; et si, député, il s'opposa à l'instauration du catholicisme en religion d'État, il se montra également fort sceptique en ce qui concerne la Fête de la Fédération, tant était grande sa peur de voir le peuple sombrer dans un nouveau cléricalisme, délit qui finit par lui valoir, ainsi qu'à son ami Destutt de Tracy, d'être emprisonné sous la Terreur. Promis à un jugement sans doute expéditif pour le I thermidor de l'an II, ils ne durent leur salut qu'aux événements du 9. Dès lors, pourquoi Volney expose-t-il sa Loi Naturelle d'abord à l'aide de la fiction du «Bon Génie » des Ruines, puis sous la forme d'un Catéchisme où il n'hésite pas à affirmer que cette loi s'origine en Dieu et que ses sectateurs ne sont pas des athées ${ }^{5}$, alors même que Les Ruines avaient montré que Dieu et les religions ne sont que des illusions dont se servent les prêtres et les grands pour asservir les peuples?

Sans doute ce délit, méthodologique et non plus social, s'explique-t-il par une des notes finales des Ruines où, s'en prenant aux « corporations ennemies de la société », c'est-à-dire aux religieux, Volney écrit :

Il serait dangereux d'attaquer de front la croyance erronée d'une nation; mais il est un art philantropique et médical de préparer les yeux à la lumière, comme les bras à la liberté. Si jamais il se forme une corporation dans ce sens, elle étonnera le monde par ses succès ${ }^{6}$.

Encore une fois, la logique de la persuasion ne se superpose pas exactement à celle de la vérité. Le chapitre XIV des Ruines ${ }^{7}$, chapitre crucial intitulé « Le grand obstacle au perfectionnement $»$, en fournit un bel exemple qui me servira de point de

5. Cf. La Loi Naturelle, chap. I, p. 447, 45 I et chap. II, p. 455.

6. Cf. Ies Ruines, note du chap. XXIII accrochèe à « Corporations ennemies de la société ». Cette phrase est absente de l'édition d'août r79ı reproduite par l'èdition du Corpus, p. 436-437

7. L'ouvrage en compte vingt-quatre. Les douze premiers exposent les principes de la loi naturelle et les causes des révolutions des empires. Les chap. XIII et XIV présentent la doctrine de la perfectibilité ou du perfectionnement. L'allégorie de la Révolution française commence au chap. XV, et le procès des religions et religieux (chap. XX-XXIV) occupe à lui seul la moitié du livre. 
départ car l'on y sent bien ce que j'appelle ici les vertus du décentrement.

Un bref flash-back sur les événements le situera. Au début du récit, le voyageur, terrassé par une méditation sur les ruines et les révolutions des empires autrefois prospères en Syrie, craint qu'il n'en advienne de même à sa chère patrie quittée en 1782 , la fatalité du « c'était écrit » semblant tout gouverner. Survient alors un Génie, un Fantôme, qui commence de lui ouvrir les yeux en lui montrant que l'homme est «l'artisan de sa destinée ${ }^{8}$, destinée heureuse s'il respecte la loi naturelle issue de l'amour de soi, garant de sa conservation grâce à ces deux sensations fondamentales que sont l'aversion de la douleur et le désir du bien-être, sensations tirant l'homme de sa barbarie et de sa sauvagerie initiales en le faisant entrer en société, laquelle, si elle entend à son tour respecter la loi naturelle, c'est-à-dire l'amour de soi, doit avoir pour principes la liberté, l'égalité et la justice, car tel est l'intérêt de tout un chacun s'il veut assurer sa propre conservation. Pour n'avoir pas respecté cette loi, et avoir laissé l'amour de soi se transformer en ignorance et en cupidité, les empires se sont tour à tour écroulés, et il en sera ainsi tant que les lois de convention ne seront pas conformes aux lois de la nature. Voilà qui ne manque pas d'abattre et de révolter un peu plus notre voyageur, d'autant plus que le Génie, dans sa maligne bonté, vient de lui montrer le conflit naissant entre les Turks et les Moscovites, car, tel est le thème du chapitre XII, « les leçons des temps passés Ise répètentl sur les temps présents ».

Faut-il donc désespérer de tout? Non, répond le Génie, qui, au chapitre XIII, soutient non seulement que l'homme est perfectible, mais encore qu'il s'est effectivement perfectionné. Le perfectionnement s'est même très nettement accéléré depuis la géniale invention de l'imprimerie, laquelle permet l'épanouissement et la diffusion de la raison, dont on perçoit enfin aujourd'hui, du côté de l'Occident, ce «bruit sourd » qu'est « le cri de la libertè ». Regardons, entendons donc qu'un siècle nouveau est en train de s'ouvrir et que la marche de la liberté et de la raison 
commence déjà de déborder les frontières de cette grande et courageuse nation : les révolutions cèdent le pas à la Révolution.

Nous voici maintenant au point en question. En effet, malgré cette belle démonstration, notre voyageur s'écrie:

O Génie. pardonne ma faiblesse : sans doute ta bouche ne peut proférer que la vérité; mais ta céleste intelligence en saisit les traits là oú mes sens grossiers ne voient que des nuages. Jen fais l'aveu : la conviction n'a point pénétré mon àme, et j'ai craint que mon doute ne te fùt une offense?

Le doute n'a rien d'un crime lui rétorque le Génie; bien au contraire, il ne faut consentir qu'à l'indubitable, et l'orateur doit supporter la contradiction, car d'elle seule naît l'évidence. Ne me crois donc pas parce que je te suis superieur, ce serait te faire violence, or « la violence est l'argument du mensonge, et imposer d'autorité une croyance est l'acte et l'indice d'un tyran $\gg^{\text {IO }}$.

Comment donc lever les doutes et donner à espérer de façon non tyrannique? Intervient ici une très subtile logique du centre et du décentrement, voire du recentrage, faisant fi de toute utopie car elle a un lieu : la France, dont il faut comprendre que la Révolution rompt avec le cycle des révolutions des empires et avec la prétendue fatalité qui le gouvernerait. Voilà ce qu'enseignent conjointement l'histoire, la géographie et la « linguistique »: « en remontant le temps, en parcourant l'espace, en comparant les langues, on apprend à relativiser les systèmes et l'on aboutit à un sain scepticisme ». Il ne reste dès lors plus qu'à anticiper quelque peu le règne du temps moderne. mais cette anticipation n'est jamais qu'une allégorie de la Révolution française et du procès qu'elle intente aux tyrans, aux nobles et aux prêtres.

Afin de dégager ces multiples lieux, véritables topoi d'un discours se voulant tout autant vrai que persuasif, observons la singulière démarche de Volney. Né en 1757, le jeune ConstantinFrançois Chassebœuf - devenu, en raison de ce ridicule patronyme, Boisgirais grâce à son père - s'établit à Paris en 1776 et s'y lie avec $\mathrm{M}^{\mathrm{me}}$ Helvétius, d'Holbach, Cabanis, puis de Tracy; peutêtre mème y fait-il la connaissance de Condillac. Il se détourne

10. Ibid. 
alors assez rapidement de ses études de médecine - études dont il semble néanmoins se souvenir lorsqu'il parle de cet « art philantropique et médical » qui se doit d'ètre une propédeutique à la fois préventive et curative- pour se consacrer aux langues orientales. En 1780, il publie un Mémoire sur la chronologie d'Hérodote qui, dit-on, suscita de belles polémiques. Mais, première originalité, la poursuite de son travail le conduit à se pencher sur l'Égypte et la Syrie et non sur les Grecs, ces faux modernes, laissant ainsi les Anciens aux anciens, c'est-à-dire aux historiens traditionnels. Troquant son nom de Boisgirais contre celui de Volney, traduction phénicienne, parait-il, de Chassebœuf, il s'embarque pour l'Égypte et la Syrie en i782 qu'en apprenant les langues locales, il parcourt tel un nomade et non tel un émir ou un prince. De retour en France trois ans plus tard, Volney publiera en i 787 son Voyage en Egypte et en Syrie.

Remarquons que le « délit méthodologique » inauguré dans le mémoire sur Hérodote s'y poursuit et s'y amplifie puisque la présentation de ces lieux, véritables berceaux de la civilisation occidentale, se veut la plus descriptive possible ainsi qu'en témoignent ces quelques lignes de la préface :

Dans ma relation, j'ai tàchè de conserver l'esprit que j'ai porté dans l'examen des faits : c'est-à-dire un amour impartial de la vérité. Je me suis interdit tout tableau d'imagination, quoique je n'ignore pas les avantages de l'illusion auprès de la plupart des lecteurs ; mais j'ai pensẽ que le genre des voyages appartenait à l'histoire et non aux romans. Je n'ai donc point représenté les pays plus beaux qu'ils ne mont paru ; je n'ai point peint les hommes meilleurs ou plus méchants que je ne les ai vus $|. .|^{11}$.

Une des principales leçons du Voyage en Egypte et en Syrie sera de rejeter le déterminisme climatique cher à Montesquieu. Volney y soutient, preuves géographiques et historiques à l'appui, l'hypothèse déjà formulée par Condillac : différentes sortes de gouvernement peuvent exister sous un même climat et sur un même sol, car ce sont précisément ces formes de gouvernement qui influent sur l'esprit des peuples ${ }^{\mathrm{I} 2}$. Leçon comparatiste et relativiste donc,

II. Cité dans Gusdorf, op, cit., p. 489.

12. Cf. Essai sur l'origine des connaissances humaines, II, i, v, $\$$ 5I et II, i, xV, $\$ \S$ I42-I45, soit p. 219 et 259 26! de l'èd. de C. Porset, Paris, Galilèe, 1973. 
mais aussi leçon sur l'histoire des langues, de l'écriture et des religions, сат le vтаi berceau, ce que montreront amplement Les Ruines, c'est l'Égypte, et le vrai drame pour les lumières, c'est le choix d'une écriture inappropriée à la diffusion de l'instruction, d'où le despotisme entraînant lui-même les révolutions des empires.

Cette dernière leçon n'est pas seulement historique, mais elle est aussi, vertu du comparatisme, politique, puisqu'elle vaut encore pour la Chine contemporaine :

Les jésuites se sont efforcés de peindre sous de belles couleurs le gouvernement chinois ; aujourd'hui l'on sait que c'est un pur despotisme oriental (entravé par le vice d'une langue) et nous donne la preuve que dans l'antiquité, jusqu'à l'invention de l'écriture alphabétique, l'esprit humain eut beaucoup de peine à se déployer, comme avant les chiffres arabes on avait beaucoup de peine à compter. Tout dépend des méthodes : on ne changera la Chine qu'en changeant sa langue ${ }^{13}$.

La réflexion historique, géographique et « linguistique » sur un lieu situé dans le temps et dans l'espace, induit donc, tant scientifiquement que politiquement, un programme et une méthode. Tout en se gardant d'ajouter ici une portée à la triste partition antirousseauiste naguère à la mode en France, il faut toutefois remarquer que Volney participe avec ses amis Idéologues à un courant de pensée s'opposant nettement à Rousseau, que ce soit sur le plan scientifique ou sur le plan politique, et ce, tant en ce qui concerne la méthode qu'en ce qui concerne le programme. Il y aurait d'ailleurs lieu de se demander si les Idéologues n'auraient pas factuellement anticipé les fortes analyses de Hegel sur la liberté et la Terreur ou sur la belle-âme. En effet, s'opposer à Robespierre et à Saint-Just ${ }^{14}$, c'était aussi s'opposer au Rousseau

13. Cf. note du chap. XIV (même observation que supra n. 6). Ce thème était fort prisé des Idéologues et je me permets de renvoyer sur ce point à J-L. Labarrière, « Le signe écrit, l'éducation et la démocratie. Quelques remarques à partir du chapitre $\mathrm{V}$ de la Grammaire de Destutt de Tracy », dans W. Busse et J. Trabant (dir.) Les Idéologues. Sémiotique, théories et politiques linguistiques pendant la Révolution française, Amsterdam, J. Benjamins, 1986, p. I67-179.

14. En plus du poids de l'Empire et de l'Université cousinienne, justement souligné par Picavet et Gusdorf, on peut se demander si l'oubli des Idéologues n'aurait pas aussi pour cause une historiographie française naguère plus tournee vers les jacobins et les montagnards plutot que vers la plaine ou les girondins, comme si 
dont ils se réclamaient, à l'anti-intellectuel chantre d'une rupestre bonté naturelle, soit aux yeux des Idéologues, autant d'ignorantins exaltant le coeur et l'enthousiasme tout en sombrant dans une misanthropie et une misologie terroristes ${ }^{\mathrm{I5}}$, faute $\mathrm{d}^{\prime}$ avoir su d'abord recueillir les faits et d'avoir ensuite su accepter ses mains, plutôt que de se résoudre, au nom d'une mythique pureté, à les rougir.

Chez Volney le lieu de l'autre, ou l'autre lieu, n'est donc ni idéalisé dans un État de Nature hors le temps et hors l'espace ni dans un peuple à venir et régénéré, qui tous deux serviraient de normes a priori pour juger des temps présents, mais il est au contraire construit a posteriori. Il faut en effet d'abord construire les

ceux-là seuls avaient été de « vais » révolutionnaires. A cela s'ajoute encore le comtisme et le kantisme de l'Université des débuts de la III République. Il est en ce sens assez amusant de constater que lorsque Maurice Blondel publiera en rgrg les leçons de Victor Delbos sur La philosophie française (Paris, Plon) - leçons de tonalité nettement « anti-boche » en raison de la Grande Guerre -, il ne pourra met tre la main sur les notes personnelles de $\mathrm{V}$. Delbos consacrées à Condillac et aux Idéologues, et devra donc se contenter de notes d'étudiants pour ce chapitre (cf. la note de Blondel, p. 250).

15. L'usage du terme « terroriste », formé en i 794 par Babeuf sur le modèle de cet autre dérivé de terreur qu'est « terrorisme », lequel date également de 1794, n'a malgré ses connotations contemporaines pas de quoi choquer. Littré le définit ainsi : «Partisan, agent du système de la terreur. Destitués sous le nom de terroristes. Babeuf, Pièces, 1, go »; le même Littré entendait d'ailleurs qu'on distingue « terrifier » de « terroriser » et qu'on réserve le second à l'établissement du terronisme, du règne de la terreur. Si donc, outre le souci du mot juste, j'emploie ici ce terme, c'est afin de souligner le lien établi par les Idéologues entre Rousseau et la Terreur, Volney n'hésitant pas à qualifier les admirateurs de Rousseau de « sectateurs tellement voisins du fanatisme qu'ils enverraient volontiers à Vincennes ceux qui n'admirent pas les Confessions ! » (cité dans Picavet, op. cit., p. I39, n. I). On se reportera sur ce point au parallèle établi par Volney entre Rousseau et Omar, parallèle valant tout autant pour les «fanatiques » de Rousseau, dans ses Leçons dHistoire, quatrième séance, p. 547548. De ce point de vue, se démarquer de Rous seau, c'ètait aussi, plus tard, s'opposer au romantisme français et allemand, courants religieux et réactionnaires aux yeux de bon nombre d'Idêologues, ainsi qu'en témoignera l'opposition constante de Volney à cet ennemi juré de l'Idéolo gie que fut Chateaubriand. Sur le sens de la Terreur et du rousseauisme en i7g4, on lira Mona Ozouf, « Guerre et Terreur dans le discours révolutionnaire : 1792 1794 », Joumal of Modern History, aut. 1984, repris dans L'école de la France, Paris, Gallimard, 1984 ; Jean-Marie Goulemot et Eric Walter, « Les centenaires de Voltaire et de Rousseau », dans Pierre Nora, Les lieux de mémoire, I. La République, Paris, Gallimard, 1984. On y verra que Louis Blanc, nettement plus rousseauiste que voltairien, n'hésitait pas à parler de terrorisme lorsque, dans son Histoire de la Révolution française (1868), il justifiait la Terreur. 
faits, mener l'enquête sur le terrain, puis comparer et si possible établir des filiations. Nous sommes ainsi plus proches des générations chères à Condillac et à Helvétius que d'une thématique de la régénération. Ne nous livrons donc pas aux hypothèses « gratuites » de ceux qui négligent les faits, mais ne cédons pas non plus à cette autorité des Anciens que croient découvrir les historiens, car ce que l'enquête apprend, c'est la variété, la diversité, la relativité, et ce qu'elle montre, c'est la négativité des temps passés, temps esclaves, temps superstitieux.

La méthode s'ouvre par là au programme : il faut accomplir une Révolution afin de rompre avec ces temps, mais, pour ce faire, il faut préalablement avoir compris du dedans l'altérité spatiotemporelle, sinon l'on reconduira ce que l'on croit rejeter. La grande leçon, toute négative, de l'histoire, est qu'elle nous montre «le tableau des folies humaines ${ }^{\sigma}$, ce qui explique d'ailleurs qu'elle ne soit pas en son lieu à l'école primaire tant elle risquerait $d^{\prime} y$ souiller la raison naissante ${ }^{17}$. En revanche nous pouvons, à l'intention des maîtres, établir, à partir des comparaisons, des filiations remontant à l'origine de l' idée de Dieu et descendant des premières croyances astronomiques égyptiennes jusqu'à la religion mahométane, dont il aurait suffi que le livre, « singé sur celui de Moïse ${ }^{18}{ }^{8}$, n'apparût point pour que dix siècles d'histoire, qui sont autant de siècles de fanatisme, eussent été changés. Or, trait méthodologique fondamental en ce qu'au nom des faits il télescope le temps et l'espace, nous avons encore sous les yeux les témoignages vivants de ces temps passés : la guerre naissante des Turcs contre les Russes, quoiqu'elle promette les Turcs à la défaite. comme l'annonce Volney dès 1788 dans ses Considérations sur la guerre des Turcs, nous montre encore aujourd'hui la nocivité des superstitions et fanatismes aveuglants et asservissants ; le despotisme chinois, organiquement lié à sa mauvaise langue et à sa mauvaise écriture, nous montre ce que furent les despotismes anciens avant l'apparition de l'alphabet, pour ne pas parler de l'invention de l'imprimerie; l'état des gouvernements des mamelouks d'Egypte et du Dey d'Alger nous montre qu'ils ne diffèrent

16. Selon le mot de Destutt de Tracy à De Gérando, cité par Gusdorf, op. cit., p. 507.

17. Cf. Leçons dHistoire, cinquième sèance, p. 559-560.

18. Cf. Leçons dHistoire, sixième séance, p. 6or. 
point essentiellement de ceux de Sparte et de Rome ; les croyances des Indiens d'Amérique, présentées dans le Voyage aux Etats-Unis, puis dans les notes et éclaircissements du Tableau du climat et du sol des Etats-Unis (1803), nous montrent qu'en matière de fatalité et de destin aveugle, ils n'ont rien à envier à Sophocle et Euripide. Le programme, aux antipodes d'une moderne attitude culturaliste, est par là tout tracé : il faut lutter contre les superstitions en faisant lire de bons livres et commencer de diffuser «ailleurs » les lumières de l'alphabet, en proposant tout d'abord une Simplification des langues orientales (I795), puis en travaillant sans relâche à la construction a posteriori d'un alphabet phonétique universel, ce qui conduira Volney à écarter la thèse monogénétique de Court de Gébelin dans son Discours sur l'étude philosophique des langues ( $18 \mathrm{ig})^{\text {I9 }}$.

Observons maintenant comment, grâce à la multiplicité des lieux spatio-temporels, méthode et programme sont à l'œuvre dans Les Ruines. L'exaltation des ruines et la sombre méditation qui s'ensuit (chap. II), précédant toutes deux l'apparition bénéfique du Fantôme (chap. III), sont nettement situées dans le temps et dans l'espace : le voyageur parcourt les ruines de Palmyre sous la onzième année du règne d'Abdül-Hamid I Ir, soit en I784, c'est-àdire peu après que les Russes se soient emparés de la Crimée. En raison même de l'objet des Ruines, qui est de réduire à néant les religions, il y a bien là un télescopage du temps et de l'espace puisque ces monuments témoignent de la richesse de ces anciens empires, lesquels étaient gouvernés par ceux qu'aussi bien les Ottomans que les Chrétiens appellent infidèles ou païens. Or, depuis que les saints et les fidèles sont au pouvoir, ils ne cessent de s'entre-tuer au nom de la vérité et laissent dépérir leurs empires. Lieux et dates ne sont donc pas innocents : le voyageur se situe en des contrées qui sont autant le berceau de notre civilisation que son tombeau puisqu'on y vit apparaître la dernière des grandes religions. La tâche du Génie, on s'en souvient, sera de montrer qu'il n'y a nulle fatalité dans la chute des empires et qu'il serait inconséquent de croire que Dieu a puni les saints alors qu'il

19. Cf. S. Auroux, Cl. Désirat et T. Hordé, « La question de l'histoire des langues et du comparatisme », Histoire, Epistémologie, Langage, 4-I, I982, p. 73-82. 
aurait autrefois béni les infidèles. Non, décidément, à conditions climatiques et géographiques constantes, il faut bien constater que l'homme est «l'artisan de sa destinee ». Afin de prouver ses dires, le Génie emmène notre voyageur dans les airs et lui ouvre les yeux et les oreilles, afin que d'un regard en surplomb - nouvelle vertu du décentrement - il puisse, tel l'historien véritable, tirer la leçon des temps passés sans rien céder à la fascination malsaine de l'autorité des Anciens, ce que Volney, chargé de la chaire d'histoire de l'École normale de l'an III (i795), où il s'opposait tant aux historiens traditionnels qu'aux nouveaux fanatiques enfin renversés, reprendra dans ces forts énoncés :

Nos ancêtres juraient par Jérusalem et la Bible, et une secte nouvelle a juré par Sparte, Athènes et Tite-Live. Ce qu'il y a de bizarre dans ce nouveau genre de religion. c'est que ses apôtres n'ont même pas eu une idée juste de la doctrine qu'ils prêchent, et que les modèles qu'ils nous ont proposés sont diamétralement contraires à leur énoncé ou à leur intention; ils nous ont vanté la liberté de Rome ou de la Grèce, et ils ont oublié qu'à Sparte une aristocratie de trente mille nobles tenait, sous un joug affreux deux cent mille serfs ; que pour empêcher la trop grande population de ce genre de négres, les jeunes Lacédémoniens allaient de nuit à la chasse aux Ilotes, comme des bêtes fauves : qu'à Athènes, ce sanctuaire de toutes les libertés, il y avait quatre têtes d'esclaves. contre une tête d'homme libre; qu'il n'y avait pas une maison ou le régime despotique de nos Colons d'Amérique ne fût exercé par ces prétendus démocrates, avec une cruauté digne de leurs tyrans; que sur environ quatre millions d'âmes qui durent peupler l'ancienne Grèce, plus de trois millions cinq cent mille étaient esclaves; que l'inégalité politique et civile était le dogme des peuples. des législateurs ; qu'il était consacré par lycurge, professé par Aristote. par le divin Platon, par les généraux et les ambassadeurs d'Athènes, de Sparte et de Rome qui, dans Polybe, dans Tite-Live, dans Thucydide parlent comme les ambassadeurs d'Attila et de Tchinguizkan ${ }^{20}$.

La fiction commencera donc par mettre en scène, sur leurs lieux mêmes, l'histoire des temps passés, et s'il y a fiction, c'est que dans Les Ruines, Volney cherche plus à convaincre qu'à faire

20. Cf. Leçons dHistoire, sixième séance, p. 603. Ce propos est cité et analysè dans la remarquable introduction de $\mathrm{P}$. Vidal-Naquet, « Tradition de la démocratie grecque », au recueil du regretté $M$. Finley, Démocratie antique et démocratie moderne, Paris, Payot, 1976. On mesurera la distance séparant Volney de Robespierre à travers l'exclamation de ce dernier : « Sparte brille comme un éclair dans des ténèbres immenses » (cité par P. Vidal- Naquet, p. 28). 
cuvre d'historien, de géographe ou de linguiste, ce qu'il fait cependant bien souvent dans ses longues notes. Il peut ainsi, contrairement à ce qui était le cas pour le Voyage en Egypte et en Syrie, chercher à frapper l'imagination du lecteur, mais, grâce au regard de plus en plus aigu que donne le Génie au voyageur, il donne en fait au lecteur le regard du bon historien. Voilà qui se vérifie très aisément dans le chapitre XII, « Leçons des temps passés répétées sur les temps présents ». On y repère en effet un basculement dans l'organisation générale des Ruines : le centre se déplace temporellement et spatialement puisque le voyageur tourne maintenant son regard du còté de la Crimée. Il y perçoit ce qui s'y déroule dans le temps présent et comprend, le Génie lui ayant de nouveau touche la vue et l'ouie, que ce qui, de haut, tant que l'on voit encore mal, semblent des insectes en train de s'agiter, ce sont bien des hommes. Or ces hommes ce sont des Tartares et des Moscovites qui se combattent, réveillant ainsi l'animosite des Musulmans envers les Russes, deux peuples se combattant au nom de Dieu, tant l'ignorance et l'esclavage en sont les véritables maîtres, ce que les réformes avortées du bref règne de Sélim III (I789-1808) con firmeront amplement, jusque dans sa déposition, puis son exécution dues à la réaction des janissaires.

Les temps présents n'ont donc en ces lieux rien changé, ce qui ne manque pas d'abattre, on s'en souvient, notre voyageur. Le Génie, philosophe et historien, soutient alors, preuves à l'appui, que l'homme est non seulement perfectible, mais qu'il s'est effectivement perfectionné, du moins en Occident. Certes, la doctrine de la perfectibilité n'a rien de particulièrement original en i7gr. Mais, ce qui l'est plus, c'est la façon dont avant la publication de l'Esquisse de Condorcet, elle est établie en en prenant pour preuve le cours de l'histoire du bassin méditerranéen, qui en vient à s'épanouir dans le temps présent sur les bords de la Seine. Ce faisant, Volney place dans la bouche du Génie une critique sans ambiguïté de cet autre tenant de la perfectibilité qu'est pour nous Rousseau :

Voudrais-tu penser que l'espece va se détériorant? Garde-toi de l'illusion et des paradoxes du misanthrope; l'homme mécontent du temps présent suppose au passé une perfection mensongère, qui n'est que le masque de son chagrin. Il loue les morts en haine des vivants, il bat les enfants avec les ossements de leurs pères. Pour 
démontrer une prétendue perfection rétrograde, il faudrait démentir le témoignage des faits et de la raison ${ }^{2 I}$.

Malheureusement un tel témoignage ne suffit pas, on s'en souvient encore, à convaincre notre voyageur. Le Génie reprend donc le voyageur рат la main et rend une fois de plus sa vue plus perçante et son ouie plus fine, afin de lui faire maintenant percevoir, quitte à anticiper quelque peu - miracle de l'écriture de qui a voyagé et entend se souvenir - l'extraordinaire Révolution qui est en train de se produire dans sa chère patrie que, cédant à « l'argument paresseux », notre voyageur serait prêt de croire vouée aux vicissitudes des révolutions. Nouveau déplacement du centre donc, puisqu'il est autant ici et maintenant que bientôt, centre dont on peut percevoir le bruit sourd jusque dans « l'espace aérien » de la Syrie. A l'initiative du peuple français, les peuples non seulement s'assemblent, se libèrent des tyrans, instituent des législateurs qui proclament une loi naturelle, c'est-àdire universelle, mais encore, répondant aux vœux du voyageur terrifié par les fanatismes religieux, instruisent le procès des religions et de leurs multiples sectes.

Le déroulement du procès ne manque pas de piment. Après une présentation des diverses religions et de leurs multiples sectes schismatiques (chap. XV), on les laisse chacune s'efforcer de «vendre leur soupe », ce qui ne manque pas d'entraîner la plus extrême violence et la plus extrême confusion entre les multiples parties en présence, mais aussi les rires et les quolibets des spectateurs amusés de tant de fadaises et d'invraisemblances (chap. XXI). La vérité n'en sort donc nullement grandie et les législateurs donnent alors la parole à un historien, fort inspiré de Dupuis, afin de remettre les choses en place. Tel est l'objet du fort long chapitre XXII. Il s'agit d'y montrer que l'idée de Dieu est issue de l'incapacité des Anciens à expliquer les bases physiques de notre univers, et que l'idée d'une vie meilleure après la mort est née de l'espoir qu'avaient les peuples asservis de se libérer un jour, illusion qu'exploitèrent prêtres et despotes pour affermir leur pouvoir. Sans développer ce point plus avant, remarquons cependant que 
la filiation entre les diverses religions est organisée de façon rigoureusement inverse entre les chapitres XXI et XXII. Lorsque la parole est donnée aux religieux, elle est d'abord donnée aux plus jeunes d'entre eux, c'est-à-dire aux musulmans, pour parvenir finalement au matérialisme taoïste, la parole passant d'une religion plus jeune à une religion plus ancienne au fur et à mesure que les diverses sectes d'une même religion s'opposent, cédant ainsi la place, en raison même de cette opposition, à une religion plus ancienne dont les partisans accusent les plus jeunes de les avoir plagiés, avant qu'ils n'en viennent eux-mêmes à s'opposer. Le temps est ainsi remonté et l'on aboutit au matérialisme unanimement rejeté par toutes les religions, d'où l'impasse.

Aussi, quand l'historien prend la parole, il part, lui, de l'origine à laquelle les religieux ont été malgré eux reconduits et il montre, étymologies à l'appui ${ }^{22}$, que ce sont les mêmes croyances, voire les mêmes dieux, qui se sont transmis jusqu'aux musulmans. Il ne reste dès lors plus à Volney qu'à achever sa démonstration en deux temps. Au chapitre XXIII, les religieux reprennent la parole et entendent s'opposer aux dires de l'historien, mais ils se déconsidèrent définitivement en s'accusant mutuellement des pires maux, avouant ainsi qu'ils n'ont cure de la vérité mais que leur seul souci est de préserver leur pouvoir en maintenant les peuples dans l'ignorance. Les législateurs, au chapitre XXIV, en concluent donc

[...] qu'il faut tracer une ligne de démarcation entre les objets vérifiables et ceux qui ne peuvent être vérifiés, et séparer d'une barrière inviolable le monde des êtres fantastiques du monde des réalités : c'est-à-dire qu'il faut ôter tout effet civil aux opinions théologiques et religieuses ${ }^{23}$.

22. Cf. Les Ruines note 81 de la page 348 : «Je sais que lon a beaucoup décrié cette recherche des étymologies : mais si, comme il est urai, les mots sont les signes représentatifs des idées, la généalogie des uns devient celle des autres, et un bon dictionnaire étymologique serait la plus parfaite histoire de l'entendement humain. Seulement il faut porter dans cette recherche des précautions que lion n'a pas prises jusqu'á ce jour, et entrautres il faut avoir fait une comparai son exacte de la valeur des lettres des divers alphabets. (p. 424).

Ajoutons que, sans pour autant faire de Volney un « génial anticipateur » de Dumézil, il est néanmoins remarquable que les notes du chap. XXII, établissant comparaisons et filiations plus encore que le corps même dudit chapitre, insistent sur ce que depuis Dumézil nous appelons la trifonctionnalité.

23. Ibid., p. 377-378. 
Les législateurs poursuivent en édictant une « religion de l'évidence et de la vérité » ${ }^{24}$ afin de satisfaire ces « hommes ignorants et pauvres $\gg^{25}$, c'est-à-dire les peuples, qui, se défiant des revendications des prêtres et trouvant l'histoire incertaine, demandent ce qu'il faut croire. Cette nouvelle religion, les législateurs l'établissent sous la forme du Catéchisme du Citoyen Français qui, en raison de son universalité, deviendra La Loi Naturelle. Or le sous-titre de cette suite et conséquence des Ruines est aussi éloquent qu'ambigu. En effet, parler de Principes Physiques de la Morale déduits de l'Organisation de lHomme et de lUnivers, c'est bien, par l'appel à la réputée très certaine physique, dresser la barrière entre le monde des êtres fantastiques et celui des réalités, mais c'est aussi supposer que les énoncés prescriptifs puissent se déduire des énoncés descriptifs. Sans s'interroger ici sur les enjeux et la pos sibilité même d'une telle déduction, demandons-nous seulement pour conclure si, au regard de son programme et de sa méthode, Volney mène à bien un tel projet.

$\mathrm{N} y$ aurait-il pas un réel « délit méthodologique » dans cette volonté qui fut la sienne d'établir un catéchisme? Est-il en effet conséquent de donner à croire là où il ne devrait y avoir que démonstration ? On pourrait certes répondre, à commencer par Volney, que c'est précisément parce que le peuple n'est encore ni en son temps ni en son lieu, qu'il faut l'éduquer sans le brusquer, d'où la fiction et la forme catéchistique. Je me demande cependant si l'on ne pourrait pas tenir le changement du titre du Catéchisme en Loi naturelle pour l'indice, aux propres yeux de Volney, d'une difficulté que, malgré ce changement, il ne parvient pas à résoudre pour au moins deux raisons. La forme catéchistique n'est pas, ainsi que le faisait déjà remarquer Condorcet, propre à une réelle instruction civique, car elle met la foi là où devrait régnet une raison critique, ce qui entraîne un désastreux sentiment d'amour envers les lois et leur confère une immuabilité et 
une autorité qu'elles ne devraient pas avoir ${ }^{26}$. Attentif à la diver sité et à la variété du réel, Volney aurait pu et dû s'aviser de ce point, mais il semble qu'au moins en sa jeunesse, il ait préféré soutenir que le réel était irrationnel en ce qu'il n'obéissait pas à sa loi. D'où la seconde raison, liée à la première, de son échec: elle tient à la volonté de fonder de façon incontestable une loi naturelle grâce au singulier renversement de l'usage métaphorique du terme « loi », qui, de l'obligation conventionnelle propre au monde politique, en est venu à définir les régularités des phénomènes du monde physique, perdant par là son caractère conventionnel, mais conservant son pouvoir d'obligation, tout se passant comme si la nature obéissait à des lois dont il faut bien alors trouver un auteur. On sait que l'oubli de la métaphore permet à une certaine forme de pensée juridique et politique de prendre les « lois » physiques pour modèles des lois politiques, et c'est à ce problème, qui dans une certaine mesure est encore aujourd'hui celui des défenseurs des Droits de l'homme, quand bien même certains ne s'en avisent guère, que Volney se trouve confronté lorsqu'il cherche à établir sa « loi naturelle», vocable ayant pour fin d'imposer au monde politique une obligation qui ne serait pas de pure convention et qui permettrait de juger des lois de convention selon qu'elles prennent ou non pour modèles les «lois » de la nature. En ce sens, c'est presque tout « naturellement » que la forme catéchistique en vient à s'imposer : afin de garantir l'universalité et l'intangibilité de cette «loi », il faut avoir recours à un anhypothétique qui, par définition, ne peut être que supposé.

De là résulte les artifices visant à soutenir que l'anhypothétique est établi à partir de l'organisation de l'univers, artifices redoublés puisqu'en plus de cet appel, on fait également appel à Dieu. Or je tiens cet appel pour n'être pas seulement un artifice rhétorique destiné à ne pas choquer le peuple, mais pour être surtout un artifice théorique destiné à masquer une réelle difficulté provenant de

26. Cf. Rapport sur ITnstruction Publique, dans Les comités d'instruction publique sous la Révolution. Principaux rapports et projets de décrets. Fascicule I. Textes revus et présentés par J. Boulad-Ayoub, M. Grenon et S. Leroux, UQAM (coll. " Recherches et Théories », N S 20), Montréal, P.U.Q., 1992, p. 36-37, 40-41 et les notes i8 et 22, p.65-67. 
ce que l'on veut à la fois trop prouver et trop obliger. On peut donc se demander si le relat if mais progressif silence politique de Volney à partir de 1795 n'aurait pas pour raison la difficulté à résoudre cette ambigu ité, raison peut-ètre plus forte que l'écœurement engendré par le Directoire, le Consulat et l'Empire. Toujours est-il qu'à partir de 1795 , les livres de Volney, plus que jamais tournès vers d'autres lieux, prennent un tour de plus en plus géographique et linguistique, comme s'il s'agissait de comprendre toujours de plus près ce qui est et non d'édicter ce qui devrait ètre. Mais, signe de ce que ni le programme ni la méthode n'ont été abandonnés, Volney attend encore de cette compréhension qu'elle permette d'agir sur les choses grâce à la diffusion des lumières. En témoigne le prix qu'en 1820 , parson testament, il institua «afin de provoqueret encourager tout travail tendant à donner suite et exécution à $\mid$ sal méthode de transcrire les langues asiatiques en langues européennes régulièrement organisées $\gg^{27}$.

\section{CNRS-EHESS}

Paris

27. Cf. Auroux, op. cit., p. 78 . Peut-être peut-on aussi tenir pour un indice de cette difficulte le fait que Volney n'ait jamais publié la suite « morale et politique » du Tableau du climat et $d u$ sol des Etats-Unis, mais se soit contenté d'y ajouter des « notes et éclaircissements ». 